\title{
Generating Multimode Entangled Microwaves with a Superconducting Parametric Cavity
}

\author{
C. W. Sandbo Chang, ${ }^{1}$ M. Simoen, ${ }^{2}$ José Aumentado, ${ }^{3}$ Carlos Sabín, ${ }^{4}$ P. Forn-Díaz, ${ }^{1}$ A. M. Vadiraj, ${ }^{1}$ \\ Fernando Quijandría, ${ }^{2}$ G. Johansson, ${ }^{2}$ I. Fuentes, ${ }^{5,6}$ and C. M. Wilson ${ }^{1, *}$ \\ ${ }^{1}$ Institute for Quantum Computing and Electrical and Computer Engineering Department, University of Waterloo, \\ 200 University Ave West, Waterloo, Ontario, N2L 3G1, Canada \\ ${ }^{2}$ MC2, Chalmers University of Technology, SE-41296, Göteborg, Sweden \\ ${ }^{3}$ National Institute of Standards and Technology, 325 Broadway, Boulder, Colorado 80305, USA \\ ${ }^{4}$ Instituto de Física Fundamental, CSIC, Serrano, 113-bis, 28006 Madrid, Spain \\ ${ }^{5}$ School of Mathematical Sciences, University of Nottingham, Nottingham NG7 2RD, United Kingdom \\ ${ }^{6}$ Faculty of Physics, University of Vienna, Boltzmanngasse 5, 1090 Vienna, Austria
}

(Received 31 August 2017; revised manuscript received 18 July 2018; published 8 October 2018)

\begin{abstract}
We demonstrate the generation of multimode entangled states of propagating microwaves. The entangled states are generated by our parametrically pumping a multimode superconducting cavity. By combining different pump frequencies, applied simultaneously to the device, we can produce different entanglement structures in a programable fashion. The Gaussian output states are fully characterized by our measuring the full covariance matrices of the modes. The covariance matrices are absolutely calibrated by our using an in situ microwave calibration source, a shot-noise tunnel junction. Applying a variety of entanglement measures, we demonstrate both full inseparability and genuine tripartite entanglement of the states. Our method is easily extensible to more modes.
\end{abstract}

DOI: 10.1103/PhysRevApplied.10.044019

\section{INTRODUCTION}

The generation and distribution of entanglement is an important problem in quantum information science. For instance, distributing entangled photons is a key paradigm in quantum communication [1]. Distributing entangled photons as a way to entangle remote processing nodes of a larger quantum computer is also a promising path toward scalability [2,3]. Multimode entangled states can also be used for a variety of quantum-networking protocols, such as quantum-state sharing [4], quantum secret sharing [5,6], and quantum-teleportation networks [7]. It is therefore of great interest to develop novel ways of efficiently generating propagating entangled states. In this work, we present a microwave circuit, a multimode parametric cavity, that generates propagating bipartite and tripartite entangled states of microwave photons. Furthermore, the entanglement structure of the tripartite states can be changed in situ by the appropriate choice of pump frequencies. The design is easily extensible to more modes using the same principle and techniques. The ability to generate complex multimode states with a programmable entanglement structure would potentially enable a number of interesting advances beyond those already mentioned,

*chris.wilson@uwaterloo.ca such as microwave cluster states [8], error-correctable logical qubits for quantum communication $[9,10]$, and the quantum simulation of relativistic quantum information processing systems $[11,12]$.

Superconducting parametric cavities have shown great promise as a quantum-technology platform in recent years. Quantum-limited parametric amplifiers have become almost commonplace in superconducting quantum computation. The parametric generation of bipartite continuousvariable (CV) entanglement between two microwave modes has been demonstrated using parametric cavities [13-16]. Other work has shown that parametric processes can coherently couple signals between different modes of a single cavity or multiple cavities, including generating superposition states of a single photon at different frequencies $[17,18]$.

In this work, by using a multimode superconducting parametric cavity, we demonstrate the generation, calibration, and verification of multimode CV entanglement, observing genuine tripartite entanglement of three propagating microwave modes. The states are fully characterized by measuring the six-by-six covariance matrix of the mode quadratures. The device operates in the steady state, functioning as a continuous source of entanglement.

The generation of multimode CV entangled states at optical frequencies has also been demonstrated in a variety 
of ways $[4,7,20-23]$. Compared with these optical implementations, our scheme is more versatile. As shown below, we can produce different entanglement structures with the same device by simply changing the frequencies of external pump tones. We can also increase the number of modes by simply adding more pump tones. In the optical experiments, these types of changes would require the time-consuming reconfiguration of an optical table.

\section{DEVICE PRINCIPLE}

The device is a quarter-wavelength coplanar waveguide resonator terminated by a superconducting quantuminterference device (SQUID) at one end (Fig. 1). At the other end, it is capacitively overcoupled $(\mathrm{Q} \approx 7000)$ to a nominally $Z_{0}=50 \Omega$ line. The device is made from Al using standard photolithography and electron-beamlithography techniques. The fundamental mode has a relatively low frequency of around $1 \mathrm{GHz}$, giving higher modes with an average frequency spacing of $2 \mathrm{GHz}$, such that three higher-order modes are accessible within our 4-8-GHz measurement bandwidth. Parametric processes are driven by a microwave pump inductively coupled to the SQUID, modulating the boundary condition of the resonator. Previous work demonstrated that this type of device could operate as a nondegenerate parametric amplifier operating near the standard quantum limit [24-26]. In a uniform cavity, the mode frequencies are equally spaced, making it difficult to address individual pairs of modes. To avoid this problem, we follow the approach of ZakkaBajjani et al. [18] and modulate the impedance of the transmission line along the length of the cavity, varying the impedance from 41 to $72 \Omega$.

As has been well documented [12,27,28], the SQUID parametrically couples the total flux in the cavity, $\hat{\Phi}_{c}$, to the pump flux, $\hat{\Phi}_{p}$, through its Hamiltonian $\hat{H}_{\text {SQUID }}=$ $E_{J}\left|\cos \left(\pi \hat{\Phi}_{p} / \Phi_{0}\right)\right| \cos \left(2 \pi \hat{\Phi}_{c} / \Phi_{0}\right)$ [29]. Starting from this relation, we can derive our interaction Hamiltonian by expanding $\hat{H}_{\text {SQUID }}$ to first order in $\hat{\Phi}_{p}$ (around the flux bias $\Phi_{\text {ext }}$ ) and to second order in $\hat{\Phi}_{c}$. Further, applying the parametric approximation to the pump, we find

$$
\hat{H}_{\mathrm{int}}=\hbar g_{0}\left(\alpha_{p}+\alpha_{p}^{*}\right)\left(\hat{a}_{1}+\hat{a}_{1}^{\dagger}+\hat{a}_{2}+\hat{a}_{2}^{\dagger}+\hat{a}_{3}+\hat{a}_{3}^{\dagger}\right)^{2},
$$

where $\alpha_{p}$ denotes the coherent pump amplitude, the bosonic operators $\hat{a}_{i}, \hat{a}_{i}^{\dagger}$ correspond to the three cavity modes considered here, and $g_{0}$ is an effective coupling constant. The internal cavity modes described by $\hat{a}_{i}$ can be connected to the propagating modes exterior to the cavity, described by operators $\hat{a}_{i, 0}$, using standard input-output theory [25].

Equation (1) contains a large number of terms corresponding to different physical processes. However, we can
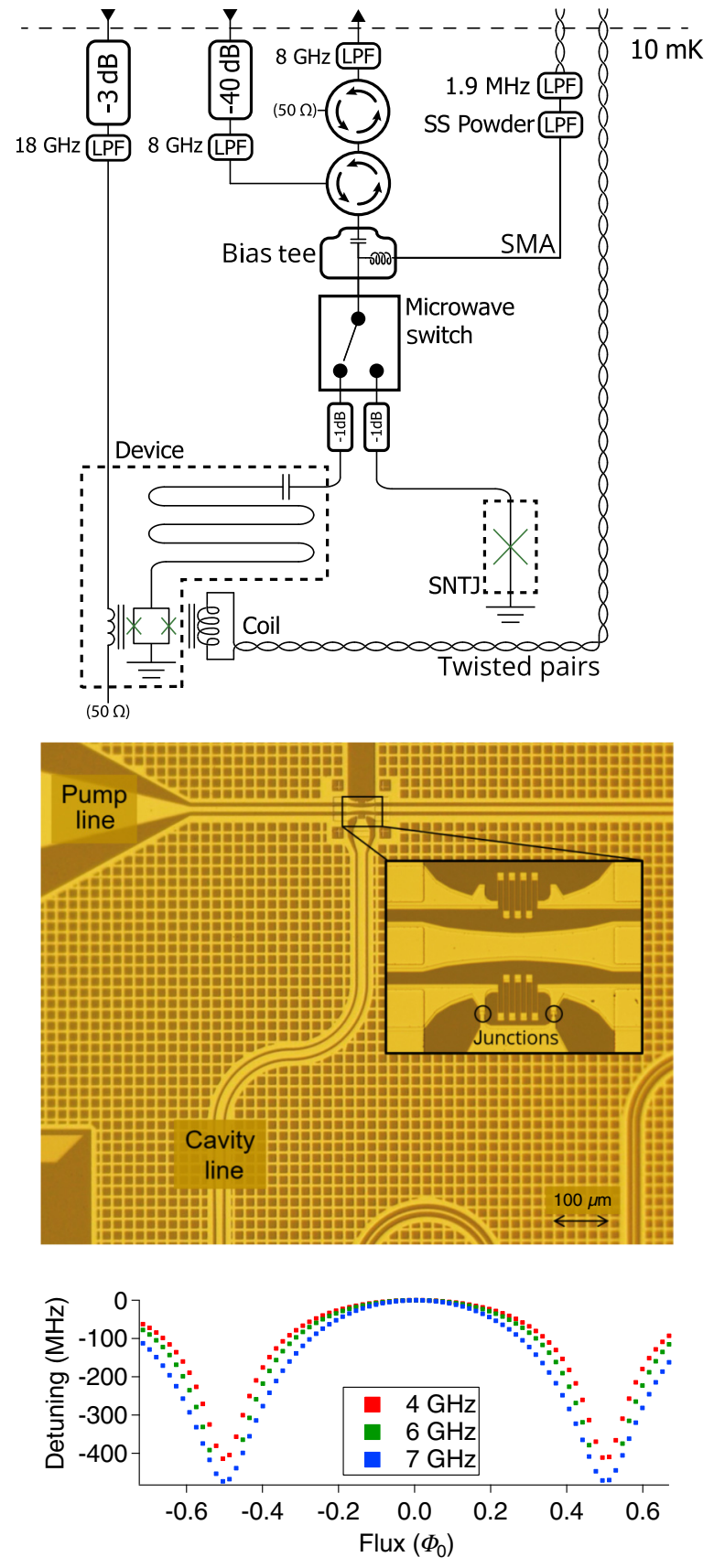

FIG. 1. Th top panel shows a simplified schematic of the measurement setup [19]. Above the bias tee, the measurement chain is shared by the cavity and the SNTJ calibration source. The short connections below the switch are made as identical as possible. The system is calibrated independently at each frequency. LPF stands for low-pass filter and SS stands for stainless steel. The middle panel shows a micrograph of the device. The inset shows an enlarged view of the SQUID. The meander in the SQUID increases the pump coupling by exploiting kinetic inductance. The bottom panel shows tuning curves of the three cavity modes, showing the detunings of the resonance frequencies with external magnetic flux, $\Phi_{\text {ext }}$ (in the unit of the flux quantum $\Phi_{0}$ ). The maximum frequencies of the three modes are $f_{1, \max }=4.217$ $\mathrm{GHz}, f_{2, \max }=6.171 \mathrm{GHz}$, and $f_{3, \max }=7.578 \mathrm{GHz}$. To allow individual difference frequencies to be addressed, the modes are dispersed by varying the cavity impedance along its length. 
selectively activate different processes by the appropriate choice of pump frequency. For instance, by choosing the sum frequency $f_{p}=f_{i}+f_{j}$, we can reduce $\hat{H}_{\text {int }}$ to $H_{\mathrm{DC}}=$ $\hbar g\left(\hat{a}_{i} \hat{a}_{j}+\hat{a}_{i}^{\dagger} \hat{a}_{j}^{\dagger}\right)$ by using the appropriate rotating-wave approximation. $H_{\mathrm{DC}}$ is well-known to produce parametric down-conversion and has been used to produce entangled photons in a wide variety of systems, including producing two-mode squeezing (TMS), a form of CV entanglement, in superconducting systems [13]. If we instead choose to pump the SQUID at the difference frequency $f_{p}=\left|f_{i}-f_{j}\right|$, $\hat{H}_{\text {int }}$ reduces to $\hat{H}_{\mathrm{CC}}=\hbar g^{\prime}\left(\hat{a}_{i} \hat{a}_{j}^{\dagger}+\hat{a}_{i}^{\dagger} \hat{a}_{j}\right)$, which produces a coherent coupling between modes.

\section{TRIPARTITE ENTANGLEMENT}

\section{A. Generation}

In this work, we demonstrate that simultaneously pumping the SQUID at two frequencies produces tripartite entanglement between modes in a way that is flexible and extensible. We consider, for instance, pumping simultaneously at $f_{\mathrm{p} 1}=f_{i}+f_{j}$ and $f_{\mathrm{p} 2}=\left|f_{k}-f_{j}\right|$. In the doubly rotating frame of the pumps, we find $\hat{H}_{\text {int }}=\hat{H}_{\mathrm{DC}}^{i, j}+\hat{H}_{\mathrm{CC}}^{j, k}$, where the superscripts refer to the modes. Because $\hat{H}_{\mathrm{DC}}^{i, j}$ and $\hat{H}_{\mathrm{CC}}^{j, k}$ do not commute, we cannot simply factor the associated time-evolution operator $\hat{U}(t)=\exp \left[-i\left(\hat{H}_{\mathrm{DC}}^{i, j}+\hat{H}_{\mathrm{CC}}^{j, k}\right) t / \hbar\right]$. However, we can find a relatively simple expression for $\hat{U}(t)$ by exploiting the symmetry properties of the Lie algebra formed by quadratic combinations of the creation and annihilation operators of the three modes. Importantly, the three operators $\hat{H}_{\mathrm{DC}}^{i, j} / \hbar g, \hat{H}_{\mathrm{CC}}^{j, k} / \hbar g^{\prime}$, and

$$
\hat{H}_{\mathrm{DC}^{\prime}}^{i, k} / \hbar g^{\prime \prime}=\left[\hat{H}_{\mathrm{DC}}^{i, j} / \hbar g, \hat{H}_{\mathrm{CC}}^{j, k} / \hbar g^{\prime}\right]=\hat{a}_{i}^{\dagger} \hat{a}_{k}^{\dagger}-\hat{a}_{i} \hat{a}_{k}
$$

form a closed subalgebra of the total algebra. Because of this, we can exactly factor $\hat{U}(t)$ into the form

$$
\hat{U}(t)=\exp \left(-i \hat{H}_{\mathrm{DC}^{\prime}}^{i, k} t / \hbar\right) \exp \left(-i \hat{H}_{\mathrm{DC}}^{i, j} t / \hbar\right) \exp \left(-i \hat{H}_{\mathrm{CC}}^{j, k} t / \hbar\right) .
$$

The effect of the simultaneous two-tone pumping is then immediately understandable. It is equivalent to applying the two pumps sequentially, but also applying a third effective pump that introduces additional correlations. This effective pump is crucial for creating tripartite entanglement. Without it, the states would at best be biseparable.

This versatile method generalizes the method first suggested in Ref. [8], where it was shown that it is theoretically possible to produce CV cluster states. Recent work has studied the computational complexity of the generated states, showing that they can be used for classically hard computations such as boson sampling [30]. The method generalizes previous work on squeezing [11], mode-mixing quantum gates [31], and entanglement $[32,33]$ in cavities undergoing relativistic motion. Earlier
TABLE I. Mode and pump frequencies for each scheme. In the CM scheme, the first pump frequency is the sum frequency of modes 1 and 2, while the second pump frequency is the difference frequency between modes 1 and 3 . In the BS scheme, the two pump frequencies are the sum frequencies of modes 1 and 2 and

\begin{tabular}{|c|c|c|}
\hline \multirow[b]{2}{*}{ Scheme } & \multicolumn{2}{|c|}{ Frequencies $(\mathrm{GHz})$} \\
\hline & Modes & Pumps \\
\hline$\overline{\mathrm{CM}}$ & $4.2039,6.1551,7.5538$ & $10.359,3.3499$ \\
\hline BS & $4.2042,6.1553,7.5545$ & $10.359,11.7587$ \\
\hline
\end{tabular}
modes 1 and 3 .

experimental work studied the development of multimode coherence in a parametric resonator pumped at two frequencies [34]. Our experimental work demonstrates that this scheme produces multimode entanglement, which has important implications for several fields, including the field of relativistic quantum information.

We present two multipartite entanglement schemes, which we call the coupled-mode (CM) scheme and the bisqueezing (BS) scheme. Both generate entanglement between three modes, but with a correlation structure that differs. In the CM scheme, described above, the device is pumped simultaneously at $f_{\mathrm{p} 1}=f_{1}+f_{2}$ and $f_{\mathrm{p} 2}=\left|f_{3}-f_{1}\right|$. The pump at $f_{\mathrm{p} 1}$ produces TMS between $f_{1}$ and $f_{2}$, while the pump at $f_{\mathrm{p} 2}$ coherently couples $f_{3}$ with $f_{1}$. In the BS scheme [35], the pump tones are applied at $f_{\mathrm{p} 1}=f_{1}+f_{2}$ and $f_{\mathrm{p} 2}=$ $f_{2}+f_{3}$, directly producing TMS correlations between the pairs. See Table I for the exact frequencies used.

\section{B. Verification}

We characterize the entanglement in our propagating output states within the covariance formalism [36]. With the good assumption that all of our $N$ modes are Gaussian [37], the state is fully characterized by the $2 N \times$ $2 N$ covariance matrix $\mathbf{V}$ of the $I$ and $Q$ voltage quadratures of the propagating modes. For the theoretical analysis, the measured voltage quadratures are calibrated and scaled, as shown below, to produce the quantities $\hat{x}_{i}=$ $\hat{a}_{i, \mathrm{o}}+\hat{a}_{i, \mathrm{o}}^{\dagger}$ and $\hat{p}_{i}=-i\left(\hat{a}_{i, \mathrm{o}}-\hat{a}_{i, \mathrm{o}}^{\dagger}\right)$. By collecting the $N$ mode quadrature operator terms into a vector operator $\hat{\mathbf{K}}=$ $\left(\hat{x}_{1}, \hat{p}_{1}, \hat{x}_{2}, \hat{p}_{2}, \ldots, \hat{x}_{N}, \hat{p}_{N}\right)^{T}$, we define the elements in $\mathbf{V}$ as $V_{i j}=\left\langle\hat{K}_{i} \hat{K}_{j}+\hat{K}_{j} \hat{K}_{i}\right\rangle / 2$, assuming the modes are mean zero.

To test the validity of our calibration, we first test if our measured covariance matrices are physical. To be physical in a classical sense, $\mathbf{V}$ has to be real, symmetric, and positive semidefinite. To be physical in the quantum sense, $\mathbf{V}$ must also obey the Heisenberg uncertainty principle. It has been shown [36] that the uncertainty principle can be expressed in terms of the symplectic eigenvalues, $v_{i}$, of $\mathbf{V}$, which are found by diagnolization of $\mathbf{V}$ through a canonical transformation of $\hat{\mathbf{K}}$. With these definitions, the 
uncertainty principle simply states $v_{i} \geq 1$ for all $i$. All the measured covariances are found to be physical [19].

We now study the entanglement properties of $\mathbf{V}$. A common measure of entanglement in $\mathrm{CV}$ systems is the logarithmic negativity, $\mathcal{N}$, which derives from the positive-partial-transpose (PPT) criterion [38,39]. The physical picture of the PPT criterion is that if we time reverse a subsystem (partition) of a multimode entangled state, then the resulting total state will be unphysical. Testing for entanglement then corresponds to confirming that the covariance matrix of the partial-transpose state $\tilde{\mathbf{V}}$ is unphysical. That is, the entanglement condition is $\tilde{v}_{\min } \equiv$ $v_{\min }(\tilde{\mathbf{V}})<1$, or equivalently $\mathcal{N} \equiv \max \left[0,-\ln \left(\tilde{v}_{\min }\right)\right]>0$.

The PPT criterion and $\mathcal{N}$ suffice to fully characterize two-mode Gaussian states, but, as is well known, classifying entanglement quickly becomes complex with increasing $N$. For three-mode states, early work suggested classifying entanglement on the basis of applying the PPT criterion to the three possible bipartitions of the state $[40,41]$. This work proposed a highest class of "fully inseparable" states, where all bipartitions are entangled. This class can be quantified by the so-called tripartite negativity $\mathcal{N}^{\text {tri }}=\left(\mathcal{N}^{A} \mathcal{N}^{B} \mathcal{N}^{C}\right)^{1 / 3}$ (where $A, B$, and $C$ label bipartitions), which is nonzero only for fully inseparable states [42].

It was later pointed out $[22,43,44]$ that although this test rules out that any one mode is separable from the whole, it does not rule out that the state is a mixture of states, each of which is separable. That is, there exist states of the form $\rho=a \rho_{1} \rho_{23}+b \rho_{2} \rho_{13}+c \rho_{3} \rho_{12}$ (where $a+b+c=1$ ), that are fully inseparable according to the above definition [43]. It was suggested that the term "genuine" tripartite entanglement be reserved for states that cannot be written as such a convex sum. This distinction between full inseparability and genuine entanglement exists only for mixed states, so understanding the purity of the state under study is important.

Teh and Reid [43] derived a set of generalized inequalities to test for genuine tripartite entanglement. We define linear combinations of our quadratures $u=h_{1} x_{1}+h_{2} x_{2}+$ $h_{3} x_{3}$ and $v=g_{1} p_{1}+g_{2} p_{2}+g_{3} p_{3}$, where the $h_{i}$ and $g_{i}$ are arbitrary real constants to be optimized. It was shown that states without genuine entanglement satisfy the inequality

$$
S \equiv\left\langle\Delta u^{2}\right\rangle+\left\langle\Delta v^{2}\right\rangle \geq 2 \min \left\{\left|h_{i} g_{i}\right|+\left|h_{j} g_{j}+h_{k} g_{k}\right|\right\},
$$

where the minimization is over permutations of $\{i, j, k\}$. We can reduce the optimization space and simplify the bound by putting restrictions on the coefficients. For this work, we use two cases: (i) $h_{l}=g_{l}=1, h_{m}=h_{n}=h$, $g_{m}=g_{n}=g, h g<1$ and (ii) $h_{l}=g_{l}=1, h_{m}=-g_{n}, h_{n}=$ $-g_{m}$, both with the search domain $[-1,1]$. With these restrictions, the bound simplifies to 2 .

\section{RESULTS}

To operate the device, the SQUID is flux biased to within $10 \%$ of $\Phi_{0}$. The pump tones are combined and feed to the on-chip pump line. The output of the device is fed through circulators to a cryogenic HEMT amplifier. After further amplification at room temperature, the

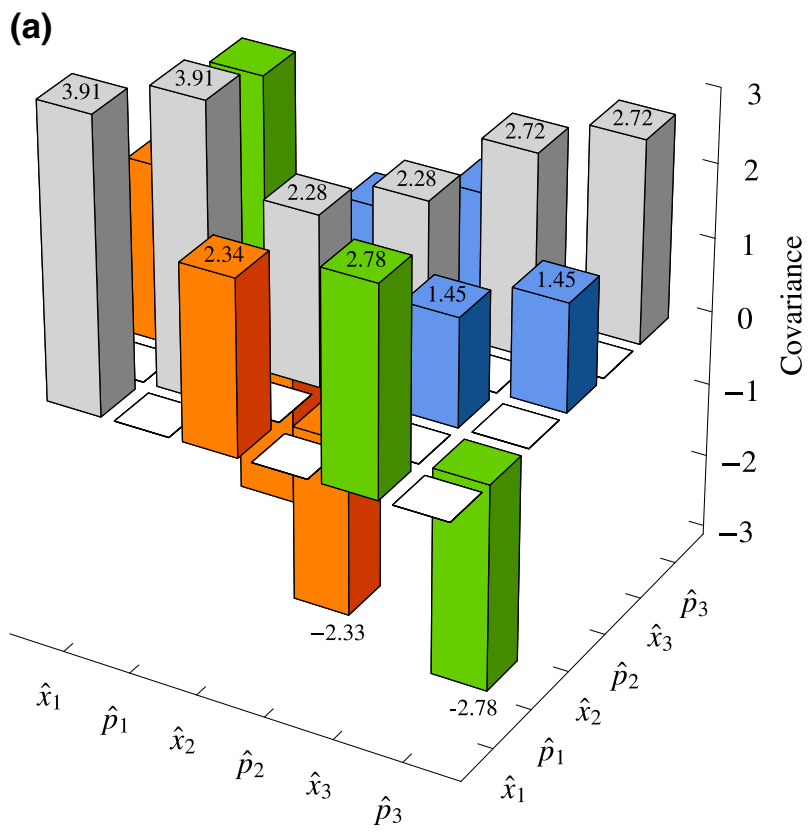

(b)

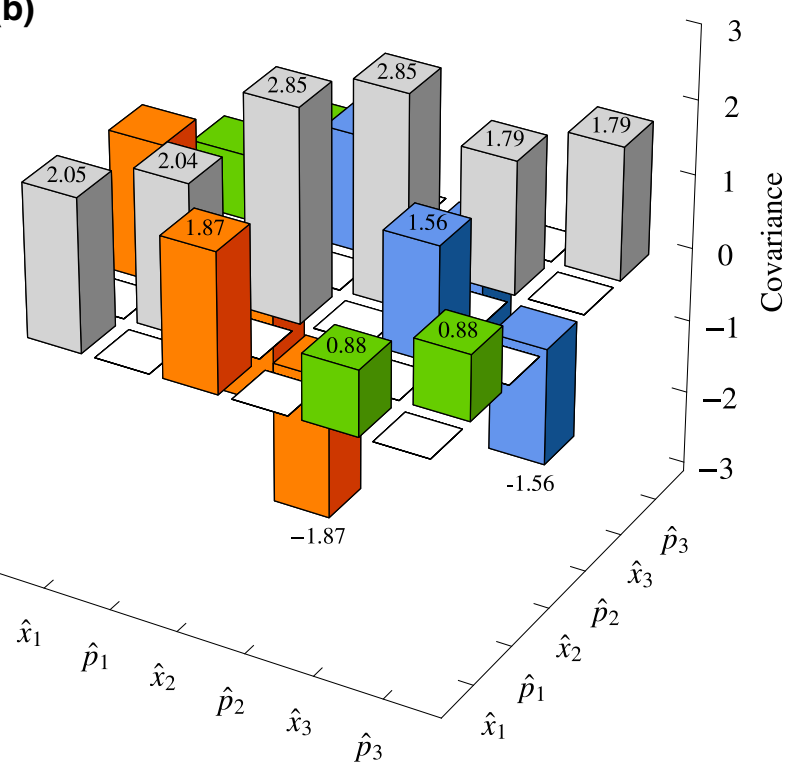

FIG. 2. Covariance matrices for our pumping schemes. Panel (a) shows the bisqueezing scheme and panel (b) shows the coupledmode scheme. As reported in Table II, these states demonstrate both full inseparability and genuine tripartite entanglement. This is the major result of this work. 
TABLE II. Entanglement measures. The $\tilde{v}_{\text {min }}$ column reports the minimum symplectic eigenvalues for all three bipartitions. The $\mathcal{N}^{\text {tri }}$ column reports the tripartite negativity. The $S$ column reports the measure of genuine tripartite entanglement in Eq. (2). The entanglement conditions are $\tilde{v}_{\min }<1, \mathcal{N}>0$, and $S<2$. Statistical errors are reported. See the Appendix for a discussion of systematic error.

\begin{tabular}{lccc}
\hline \hline Scheme & $\tilde{v}_{\min }$ & $\mathcal{N}^{\text {tri }}$ & $S$ \\
\hline CM & $0.48 \pm 0.002,0.39 \pm 0.002,0.57 \pm 0.002$ & $0.73 \pm 0.005$ & $1.49 \pm 0.01$ \\
BS & $0.31 \pm 0.003,0.48 \pm 0.004,0.39 \pm 0.004$ & $0.94 \pm 0.012$ & $1.19 \pm 0.01$ \\
\hline \hline
\end{tabular}

signal is split in two paths and then fed through custommade image-rejection filters into a pair of rf digitizers. The digitizers output $I$ and $Q$ samples. In this work, the bandwidth is $1 \mathrm{MHz}$. The measurements are done sequentially for the three mode pairs. The covariance matrix of the $I$ and $Q$ time series is then computed. We remove the effects of drift by performing a chopped measurement, with the pumps turned on and off for $5 \mathrm{~s}$ each. The differenced data are then averaged over many cycles, typically 1000 .

The entanglement tests described above compare the variances and covariances of the modes at the level of the vacuum noise. It is therefore essential to have an accurate, absolute calibration of $\mathbf{V}$. In this experiment, we perform this calibration using a shot-noise tunnel junction (SNTJ) $[45,46]$ produced by the National Institute of Standards and Technology in Boulder. See the Appendix for the details of the calibration [19].

The quadrature voltages at room temperature, $\hat{I}_{i}$ and $\hat{Q}_{i}$, are converted to the scaled quadrature variables $\hat{x}_{i}$ and $\hat{p}_{i}$ with use of the calibrated system gains, $G_{i}$. Following recent work [14], the scaled variance at the device output is

$$
\left\langle\hat{x}_{i}^{2}\right\rangle=\frac{4\left(\left\langle\hat{I}_{i}^{2}\right\rangle_{\text {on }}-\left\langle\hat{I}_{i}^{2}\right\rangle_{\text {off }}\right)}{G_{i} Z_{0} h f_{i} B}+\operatorname{coth} \frac{h f_{i}}{2 k_{B} T_{i}},
$$

where $B$ is the measurement bandwidth, with a similar definition for $\hat{p}_{i}$. The hyperbolic cotangent term here represents the input quantum noise, at temperature $T_{i}$, which is (unfortunately) subtracted when we subtract the reference noise measured with the pump off. Without the input noise, the output variance will be underestimated, leading to an overestimate of the degree of entanglement or even an erroneous claim of entanglement. It is therefore critical to characterize $T_{i}$ [47]. Assuming the mode is in the vacuum state is tantamount to assuming that the system is entangled. In our setup, the calibration of the system gain using the SNTJ also gives us the physical electron temperature of the SNTJ. As detailed in the Appendix and Ref. [19], we find values of $25-37 \mathrm{mK}$. For our working frequencies, these temperatures are deeply in the quantum regime, giving $\operatorname{coth}\left(h f_{i} / 2 k_{B} T_{i}\right)=1.00$ with at least three significant figures.

Estimating the covariances of our modes is easier since neither the input noise nor the system noise is correlated at different frequencies. We then obtain the covariance by simply rescaling the room-temperature values as, for example,

$$
\left\langle\hat{x}_{i} \hat{x}_{j}\right\rangle=\frac{4\left\langle\hat{I}_{i} \hat{I}_{j}\right\rangle_{\mathrm{on}}}{\sqrt{G_{i} G_{j} f_{j} f_{j}} Z_{0} h B} .
$$

As $\mathbf{V}$ is symmetric, just 21 terms in the matrix need to be individually measured for $N=3$ modes.

The results of our measurements are shown in Fig. 2. As shown in Table II, we find that the states generated by the two schemes show both full inseparability and genuine tripartite entanglement.

The main limitation on the degree of entanglement in the system seems to be the purity of the states. For an ideal system, pumping harder should increase the degree of squeezing and entanglement together. We instead see that the squeezing increases, but the purity of the states simultaneous declines, limiting the maximum degree of entanglement. This suggests a nonideality such as higher-order nonlinearities or parasitic coupling to other modes.

\section{ACKNOWLEDGMENTS}

We thank B. Plourde, J.J. Nelson, and M. Hutchings at Syracuse University for invaluable help in junction fabrication. We also acknowledge M. Piani for helpful discussions. C.M.W., C.W.S.C., P.F.-D., and A.M.V. acknowledge NSERC of Canada, the Canadian Foundation for Innovation, the Ontario Ministry of Research and Innovation, Canada First Research Excellence Fund, Industry Canada, and the $\mathrm{CMC}$ for financial support. C.S. acknowledges financial support by Fundación General CSIC (Programa ComFuturo), MINECO/FEDER Grant No. FIS2015-70856-P, and CAM PRICYT Project QUITEMAD+ Grant No. S2013/ICE-2801. I.F. acknowledges EPSRC (Career Acceleration Fellowship Grant No. EP/G00496X/2). F.Q. and G.J. acknowledge the Knut and Alice Wallenberg Foundation for funding.

\section{APPENDIX: CALIBRATION}

The SNTJ is an $\mathrm{Al} / \mathrm{Al}$ oxide/Al tunnel junction packaged with a rare-earth magnet that quenches superconductivity in the Al. It is fabricated to have a normal-state resistance, $R_{n}$, very close to $50 \Omega$ such that any noise it generates is well coupled to the transmission line. The output noise power of the voltage-biased SNTJ coupled to a 


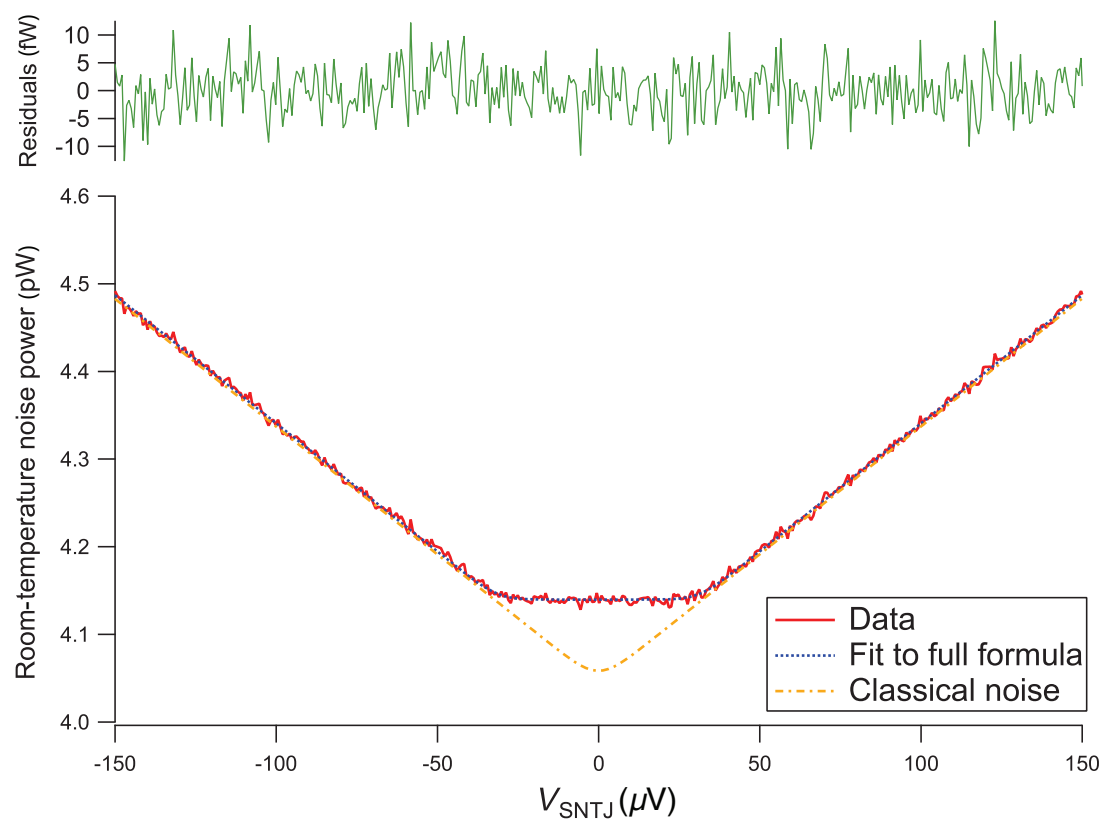

FIG. 3. Fit of the noise power from the SNTJ, measured at room temperature. The noise power (solid red line) is shown as a function of the SNTJ bias voltage. The theory curve (dotted blue line) shows the fit to Eq. (A1), from which we extract the system gain, $G$, the physical electron temperature, $T$, and the system noise temperature, $T_{N}$. The green curve shows the residuals of the fit. The flatness of the zero-voltage power is a strong qualitative indication that the system is in the quantum regime $\left(h f \gg k_{B} T\right)$. For comparison, we show the corresponding "classical" curve (dash-dotted orange line), with the frequency set to $f=0$, such that the vacuum-noise contribution is zero. impedance-matched measurement system is $[45,46]$

$$
\begin{aligned}
P_{\mathrm{RT}}= & G B k_{B}\left\{T_{N}+\frac{1}{2}\left[\left(\frac{\mathrm{eV}+\mathrm{hf}}{2 k_{B}}\right) \operatorname{coth}\left(\frac{\mathrm{eV}+\mathrm{hf}}{2 k_{B} T}\right)\right.\right. \\
& \left.\left.+\left(\frac{\mathrm{eV}-\mathrm{hf}}{2 k_{B}}\right) \operatorname{coth}\left(\frac{\mathrm{eV}-\mathrm{hf}}{2 k_{B} T}\right)\right]\right\},
\end{aligned}
$$

where $G$ is the system gain, $B$ is the measurement bandwidth, $V$ is the voltage across the SNTJ, $T$ is the physical electron temperature of the SNTJ, and $T_{N}$ is the system noise temperature. This fundamental expression includes both the quantum Johnson noise around zero voltage and the shot noise for larger voltages. Measuring $P_{\mathrm{RT}}$ as a function of $V$ allows us to extract the unknown parameters in our system: $G, T$, and $T_{N}$. Roughly speaking, the shot noise of the junction for large $V$ provides a known power that allows us to calibrate $G$. With $G$ known, we can then extract the absolute power level at small biases. In the classical limit $\left(k_{B} T \gg h f\right)$, this small-bias power gives us $T$. Note that $T_{N}$ is not a physical temperature in the system, but expresses the noise added to the measurement by the amplification chain in the unit of temperature (referred to the SNTJ junction).

To perform the calibration, an $f_{\text {bias }}=1 \mathrm{kHz}$ triangle wave is applied to the dc bias circuit at room temperature. (See Ref. [19] for more details of the bias circuit.) The corresponding voltage applied to the SNTJ is $V_{\mathrm{SNTJ}}=$ $361 \mu \mathrm{V}$ peak to peak. This corresponds to an amplitude of approximately $7 \times h(6 \mathrm{GHz}) / e$.

With the triangle wave applied, the output noise power is measured as a function of time at room temperature. The values of $V_{\text {SNTJ }}=361 \mu V$ peak to peak and $f_{\text {bias }}=1 \mathrm{kHz}$ are used to scale the $x$ axis from time to voltage before we fit the measured data to Eq. (A1) (see Fig. 3). From the fit, we can extract the unknown parameters of our system: $G, T$, and $T_{N}$ (Table III). Although the full Eq. (A1) is used to fit the whole curve, the different parameters can largely be estimated independently from different parts of the curve. For large $V \gg h f / e$, the noise power is dominated by shot noise, which has a linear voltage dependence and a well-known absolute power of $2 \mathrm{eV} / R_{n}$. Measuring the slope of the linear part of the curve therefore allows us to calibrate $G$. With $G$ known, the power levels can be scaled absolutely to the plane of the SNTJ. Since the shot-noise contribution goes to zero for $V=0$, the $y$ intercept gives the added noise, $T_{N}$. The difference between $T_{N}$ and the total noise measured at $V=0$ is then the equilibrium noise added by the unbiased SNTJ (i.e., the quantum Johnson noise, which includes contributions from thermal Johnson noise and the vacuum noise of the junction resistance). In the classical regime $\left(k_{B} T \gg h f\right)$, the noise is dominated by Johnson noise, and the noise power directly gives the physical electron temperature, $T$. In the quantum regime $\left(h f \gg k_{B} T\right)$, the zero-bias noise level is dominated by vacuum noise and becomes insensitive to temperature. However, the curvature of the noise power near $2 \mathrm{eV} \pm h f \sim k T$ (where it transitions between the flat-bottomed quantum-noise and the linear shot-noise dependence), still yields $T$, although with less precision. Both regimes are fully contained in Eq. (A1), and fitting is always done to the full formula. An example fit is shown in Fig. 3. Using the measurement frequency $f=7.5538$ $\mathrm{GHz}$, we obtain $G=45.6 \mathrm{~dB}, T=25.8 \mathrm{mK}$, and $T_{N}=$ $8.03 \mathrm{~K}$. (See Ref. [19] for full calibration results, including error estimates.) The flatness of the curve near zero voltage is a strong qualitative indication that the system is in the quantum regime $h f \gg k_{B} T$. For comparison, we show the corresponding "classical" curve, with the frequency set 
TABLE III. Calibration results. Each mode is calibrated twice, before the three mode-pair combinations $\{1|2,1| 3,2 \mid 3\}$ are measured. The results of both calibrations are shown. The calibrations are done consecutively over a span of a few hours. We observe a drift in system gain of about $1 \%$. The input temperature ranges from 25 to $37 \mathrm{mK}$ throughout all calibrations done. This level of drift in the physical temperature of the cryostat is not unusual. Further, it is easily verified that for all combinations of frequency and temperature, we are deeply in the quantum regime with $\operatorname{coth}\left(h f_{i} / 2 k_{B} T_{i}\right)=1.00$ to at least three significant figures. It is because of this that we have a relatively large uncertainty in the measured temperature, of order $10 \%$, even the though the uncertainty in the measured noise power, dominated by quantum noise, is much smaller.

System gain

\begin{tabular}{lccccccc} 
& \multicolumn{3}{c}{ Frequency $(\mathrm{GHz})$} & & \multicolumn{3}{c}{ System gain/1000 } \\
\cline { 2 - 4 } & Mode 1 & Mode 2 & Mode 3 & & Mode 1 & Mode 2 & Mode 3 \\
\hline CM & 4.2039 & 6.1551 & 7.5538 & & $2461 \pm 3,2485 \pm 3$ & $138.2 \pm 0.18,90.6 \pm 0.12$ & $36.8 \pm 0.06,36.5 \pm 0.06$ \\
BS & 4.2042 & 6.1553 & 7.5545 & & $2455 \pm 3,2450 \pm 3$ & $136.8 \pm 0.17,89.0 \pm 0.13$ & $37.1 \pm 0.06,36.4 \pm 0.06$ \\
\hline
\end{tabular}

Input temperature

Frequency $(\mathrm{GHz})$

$T_{\text {in }}(\mathrm{mK})$

\begin{tabular}{lccccccc} 
& Mode 1 & Mode 2 & Mode 3 & Mode 1 & Mode 2 & Mode 3 \\
\hline CM & 4.2039 & 6.1551 & 7.5538 & & $30.0 \pm 3,32 \pm 3.1$ & $25.6 \pm 3.2,27 \pm 3.2$ & $32.1 \pm 3.6,25.8 \pm 3.8$ \\
BS & 4.2042 & 6.1553 & 7.5545 & $29 \pm 3.1,25.7 \pm 3.2$ & $28.2 \pm 3,32.2 \pm 3.2$ & $37.3 \pm 3.4,29.6 \pm 3.6$ \\
\hline
\end{tabular}

System noise temperature

\begin{tabular}{lccccccc} 
& \multicolumn{3}{c}{ Frequency $(\mathrm{GHz})$} & & \multicolumn{2}{c}{$T_{\mathrm{N}}(\mathrm{K})$} \\
\cline { 2 - 3 } \cline { 6 - 7 } & Mode 1 & Mode 2 & Mode 3 & Mode 1 & Mode 2 & Mode 3 \\
\hline CM & 4.2039 & 6.1551 & 7.5538 & & $6.07 \pm 0.009,6.07 \pm 0.009$ & $6.28 \pm 0.009,6.05 \pm 0.009$ & $8.03 \pm 0.014,8.03 \pm 0.014$ \\
BS & 4.2042 & 6.1553 & 7.5545 & & $6.07 \pm 0.009,6.07 \pm 0.008$ & $6.30 \pm 0.010,6.08 \pm 0.008$ & $7.98 \pm 0.014,8.05 \pm 0.014$ \\
\hline \hline
\end{tabular}

to $f=0$, such that the vacuum-noise contribution is zero. The difference is clear.

Figure 3 shows the calibration result for the coupledmode scheme for digitizer 2 and mode 3 . For each scheme, the calibration is performed twice at each frequency, once for each mode pairing in the covariance matrix $\{1|2,1| 3,2 \mid 3\}$. As the signal is split at room temperature to feed two microwave digitizers, they have to be calibrated individually to ensure that any imbalance in the two systems is removed. Therefore, for each tripartite entanglement experiment, a set of six calibrations is performed.

As a way to estimate the overall systematic error in the calibration, we can measure the output of the same mode with the two digitizers simultaneously. That is, if there were no systematic error in the system, the measured values of the noise power referred to the output of the cavity should agree perfectly. We instead find that they disagree by $0.5 \%$ for the different modes. This systematic error is comparable to the random error in our measurements.

[1] Thomas Jennewein, Christoph Simon, Gregor Weihs, Harald Weinfurter, and Anton Zeilinger, Quantum cryptography with entangled photons, Phys. Rev. Lett. 84, 4729 (2000).

[2] H. J. Kimble, The quantum internet, Nature 453, 1023 (2008).

[3] S. Felicetti, M. Sanz, L. Lamata, G. Romero, G. Johansson, P. Delsing, and E. Solano, Dynamical Casimir effect entangles artificial atoms, Phys. Rev. Lett. 113, 793 (2014).
[4] Andrew M. Lance, Thomas Symul, Warwick P. Bowen, Barry C. Sanders, and Ping Koy Lam, Tripartite quantum state sharing, Phys. Rev. Lett. 92, 4 (2004).

[5] Richard Cleve, Daniel Gottesman, and Hoi-Kwong Lo, How to share a quantum secret, Phys. Rev. Lett. 83, 648 (1999).

[6] Tomás Tyc, and Barry C. Sanders, How to share a continuous-variable quantum secret by optical interferometry, Phys. Rev. A 65, 612 (2002).

[7] H. Yonezawa, T. Aoki, and A. Furusawa, Demonstration of a quantum teleportation network for continuous variables, Nature 431, 430 (2004).

[8] D. E. Bruschi, C. Sabín, P. Kok, G. Johansson, P. Delsing, and Ivette Fuentes, Towards universal quantum computation through relativistic motion, Sci. Rep. 6, 18349 (2016).

[9] A. Bolt, G. Duclos-Cianci, D. Poulin, and T. M. Stace, Foliated quantum error-correcting codes, Phys. Rev. Lett. 117, 721 (2016).

[10] Paul Jouguet, Sébastien Kunz-Jacques, Anthony Leverrier, Philippe Grangier, and Eleni Diamanti, Experimental demonstration of long-distance continuous-variable quantum key distribution, Nat. Photonics 7, 378 (2013).

[11] David Edward Bruschi, Andrzej Dragan, Antony R. Lee, Ivette Fuentes, and Jorma Louko, Relativistic motion generates quantum gates and entanglement resonances, Phys. Rev. Lett. 111, 090504 (2013).

[12] C. M. Wilson, G. Johansson, A. Pourkabirian, M. Simoen, J. R. Johansson, T. Duty, F. Nori, and P. Delsing, Observation of the dynamical Casimir effect in a superconducting circuit, Nature 479, 376 (2011).

[13] E. Flurin, N. Roch, F. Mallet, M. H. Devoret, and B. Huard, Generating entangled microwave radiation over 
two transmission lines, Phys. Rev. Lett. 109, 183901 (2012).

[14] E. Flurin, N. Roch, J.-D. Pillet, F. Mallet, and B. Huard, Superconducting quantum node for entanglement and storage of microwave radiation, Phys. Rev. Lett. 114, 090503 (2015).

[15] Kirill G. Fedorov, L. Zhong, S. Pogorzalek, P. Eder, M. Fischer, J. Goetz, E. Xie, F. Wulschner, K. Inomata, T. Yamamoto, Y. Nakamura, R. Di Candia, U. Las Heras, M. Sanz, E. Solano, E. P. Menzel, F. Deppe, A. Marx, and R. Gross, Displacement of propagating squeezed microwave states, Phys. Rev. Lett. 117, 020502 (2016).

[16] Kirill G. Fedorov, S. Pogorzalek, U. Las Heras, M. Sanz, P. Yard, P. Eder, M. Fischer, J. Goetz, E. Xie, K. Inomata, Y. Nakamura, R. Di Candia, E. Solano, A. Marx, F. Deppe, and R. Gross, Finite-time quantum entanglement in propagating squeezed microwaves, Sci. Rep. 8, 6416 (2018).

[17] A. J. Sirois, M. A. Castellanos-Beltran, M. P. DeFeo, L. Ranzani, F. Lecocq, R. W. Simmonds, J. D. Teufel, and J. Aumentado, Coherent-state storage and retrieval between superconducting cavities using parametric frequency conversion, Appl. Phys. Lett. 106, 172603 (2015).

[18] Eva Zakka-Bajjani, Franćois Nguyen, Minhyea Lee, Leila R. Vale, Raymond W. Simmonds, and José Aumentado, Quantum superposition of a single microwave photon in two different 'colour' states, Nat. Phys. 7, 599 (2011).

[19] See Supplemental Material at http://link.aps.org/supple mental/10.1103/PhysRevApplied.10.044019 for more details on the calibration and error analysis.

[20] Takao Aoki, Nobuyuki Takei, Hidehiro Yonezawa, Kentaro Wakui, Takuji Hiraoka, Akira Furusawa, and Peter van Loock, Experimental creation of a fully inseparable tripartite continuous-variable state, Phys. Rev. Lett. 91, 080404 (2003).

[21] Matthew Pysher, Yoshichika Miwa, Reihaneh Shahrokhshahi, Russell Bloomer, and Olivier Pfister, Parallel generation of quadripartite cluster entanglement in the optical frequency comb, Phys. Rev. Lett. 107, 030505 (2011).

[22] S. Gerke, J. Sperling, W. Vogel, Y. Cai, J. Roslund, N. Treps, and C. Fabre, Multipartite entanglement of a twoseparable state, Phys. Rev. Lett. 117, 773 (2016).

[23] L. K. Shalm, D. R. Hamel, Z. Yan, C. Simon, K. J. Resch, and T. Jennewein, Three-photon energy-time entanglement, Nat. Phys. 9, 19 (2012).

[24] M. Simoen, C. W. S. Chang, P. Krantz, Jonas Bylander, W. Wustmann, V. Shumeiko, P. Delsing, and C. M. Wilson, Characterization of a multimode coplanar waveguide parametric amplifier, J. Appl. Phys. 118, 154501 (2015).

[25] Waltraut Wustmann, and Vitaly Shumeiko, Parametric resonance in tunable superconducting cavities, Phys. Rev. B 87, 184501 (2013).

[26] Waltraut Wustmann, and Vitaly Shumeiko, Non-degenerate parametric resonance in tunable superconducting cavity, Phys. Rev. Appl. 8, 024018 (2017).

[27] T. Yamamoto, K. Inomata, M. Watanabe, K. Matsuba, T. Miyazaki, William D. Oliver, Yasunobu Nakamura, and J. S. Tsai, Flux-driven Josephson parametric amplifier, Appl. Phys. Lett. 93, 042510 (2008).

[28] C. M. Wilson, T. Duty, M. Sandberg, F. Persson, V. Shumeiko, and P. Delsing, Photon generation in an electromagnetic cavity with a time-dependent boundary, Phys. Rev. Lett. 105, 233907 (2010).

[29] J. R. Johansson, G. Johansson, C. M. Wilson, and Franco Nori, Dynamical Casimir effect in a superconducting coplanar waveguide, Phys. Rev. Lett. 103, 147003 (2009).

[30] Borja Peropadre, Joonsuk Huh, and Carlos Sabín, Dynamical Casimir effect for gaussian boson sampling, Sci. Rep. 8, 3751 (2018).

[31] D. E. Bruschi, J. Louko, D. Faccio, and Ivette Fuentes, Mode-mixing quantum gates and entanglement without particle creation in periodically accelerated cavities, New J. Phys. 15, 073052 (2013).

[32] Nicolai Friis, David Edward Bruschi, Jorma Louko, and Ivette Fuentes, Motion generates entanglement, Phys. Rev. D 85, 1 (2012).

[33] Nicolai Friis and Ivette Fuentes, Entanglement generation in relativistic quantum fields, J. Mod. Opt. 60, 22 (2013).

[34] Gheorghe Sorin Paraoanu, Juha Hassel, Pasi Lahteenmaki, and Pertti J. Hakonen, Coherence and multimode correlations from vacuum fluctuations in a microwave superconducting cavity, Nat. Commun. 7, 12548 (2016).

[35] David Edward Bruschi, Carlos Sabń, and Gheorghe Sorin Paraoanu, Entanglement, coherence, and redistribution of quantum resources in double spontaneous down-conversion processes, Phys. Rev. A 95, 062324 (2017).

[36] R. Simon, N. Mukunda, and Biswadeb Dutta, Quantumnoise matrix for multimode systems: $U(n)$ invariance, squeezing, and normal forms, Phys. Rev. A 49, 1567 (1994).

[37] Using the standard Fisher-Pearson skewness coefficient, we find that the skew (third moment) of our experimental data is not statistically significant, supporting the Gaussian nature of the states.

[38] R. Simon, Peres-Horodecki separability criterion for continuous variable systems, Phys. Rev. Lett. 84, 2726 (2000).

[39] Gerardo Adesso and Fabrizio Illuminati, Gaussian measures of entanglement versus negativities: Ordering of two-mode Gaussian states, Phys. Rev. A 72, 032334 (2005).

[40] G. Giedke, B. Kraus, M. Lewenstein, and J. I. Cirac, Separability properties of three-mode Gaussian states, Phys. Rev. A 64, 052303 (2001).

[41] Peter van Loock, and Akira Furusawa, Detecting genuine multipartite continuous-variable entanglement, Phys. Rev. A 67, 052315 (2003).

[42] C. Sabín and G. García-Alcaine, A classification of entanglement in three-qubit systems, Eur. Phys. J. D 48, 435 (2008).

[43] R. Y. Teh, and M. D. Reid, Criteria for genuine N-partite continuous-variable entanglement and Einstein-PodolskyRosen steering, Phys. Rev. A 90, 062337 (2014).

[44] E. Shchukin, and P. van Loock, Generalized conditions for genuine multipartite continuous-variable entanglement, Phys. Rev. A 92, 042328 (2015).

[45] L. Spietz, K. W. Lehnert, I. Siddiqi, and R. J. Schoelkopf, Primary electronic thermometry using the shot noise of a tunnel junction, Science 300, 1929 (2003).

[46] Lafe Spietz, R. J. Schoelkopf, and Patrick Pari, Shot noise thermometry down to $10 \mathrm{mK}$, Appl. Phys. Lett. 89, 183123 (2006).

[47] D. E. Bruschi, N. Friis, I. Fuentes, and S. Weinfurtner, On the robustness of entanglement in analogue gravity systems, New J. Phys. 15, 113016 (2013). 